\title{
General Zakharov-Shabat Equations, Multi-Time Hamiltonian Formalism, and Constants of Motion
}

\author{
L. A. Dickey* \\ Department of Mathematics, Brandeis University, Waltham, MA 02254, USA
}

\begin{abstract}
We construct a Hamiltonian formalism for general Zakharov-Shabat equations (zero curvature equations with rational dependence on a parameter) as well as their constants of motion, and prove that the latter are in involution. The field-theoretical (multi-time) Hamiltonian formalism is used.
\end{abstract}

\section{Introduction}

As soon as the complete integrability of the $\mathrm{KdV}$ equation was discovered it was also found that this equation was a Hamiltonian system. Later on Hamiltonian structures were suggested for all hierarchies of integrable equations: for generalized $\mathrm{KdV}$, for KP, AKNS etc. However this was not done for the most general scheme for obtaining integrable systems given by Zakharov and Shabat. They considered a zero curvature equation with a rational dependence on a spectral parameter.

Here we construct a Hamiltonian structure for these equations. We have a typical case when a field-theoretical (or multi-time) formalism is natural since both independent variables are equal by right and there is no reason to prefer one of them to the other as a time variable in which the system evolves. It is enough to know a Lagrangian, and then all the elements of the formalism are recovered automatically. Our construction of the Lagrangian generalizes that given by Zakharov and Mikhailov [8] for the case of simple poles.

The Lagrange-Hamiltonian field-theoretical formalism is well-known. However, the specific form we use fits very well to integrable systems under consideration. It has a formal-algebraic character and admits easy calculations. Briefly it was described in a lecture [11] (an earlier sketch in [4]). A complete description is in [3].

\footnotetext{
* Supported by National Science Foundation, Research Grant DMS 8703407. Address after January 1990: Mathematics Department, University of North Carolina, Chapel Hill, ṆC 27514, USA
} 


\section{Equations}

Let us consider the zero-curvature equations (see [1])

$$
U_{\eta}-V_{\xi}=[U, V] \text {, }
$$

where $U$ and $V$ are matrix meromorphic functions of a variable $z$ :

$$
\begin{aligned}
U & =U_{0}+\sum_{k=1}^{N_{1}} U_{k}, & V & =V_{0}+\sum_{k=1}^{N_{2}} V_{k}, \\
U_{k} & =\sum_{r=0}^{n_{k}} U_{k, r}\left(z-a_{k}\right)^{-r-1}, & V_{k} & =\sum_{r=0}^{m_{k}} V_{k, r}\left(z-b_{k}\right)^{-r-1} .
\end{aligned}
$$

The equations must hold identically with respect to $z$, the poles $a_{k}$ and $b_{k}$ are constant. Some of $a_{k}$ may coincide with some of $b_{l}$; without loss of generality we can assume that in this case they have the same subscripts, i.e. $a_{k} \neq b_{l}$ if $k \neq l$. The resulting equations for $\left\{U_{k}, V_{k}\right\}$ are called Zakharov-Shabat equations.

It is easy to see that in order to prove that Eq. 1 holds it suffices to verify that the principal parts of the left-hand side and of the right-hand side coincide in all the poles and that the limits of the left-hand side and of the right-hand side when $z \rightarrow \infty$ are equal, i.e.

$$
U_{0 \eta}-V_{0 \xi}=\left[U_{0}, V_{0}\right] \text {. }
$$

The latter equation implies the existence of a matrix $t$ such that

$$
U_{0}=t^{-1} t_{\xi}, \quad V_{0}=t^{-1} t_{\eta} .
$$

An elementary computation shows that the number of equations is less by 1 than the number of unknown matrices, i.e. one of the matrices can be chosen freely. Moreover even after this choice there remains freedom of choice of a number of diagonal matrices (like in a matrix equation $[A, X]=0$ where $X$ is unknown: in the basis where $A$ is diagonal $X$ may be an arbitrary diagonal matrix), a detailed analysis of a special case see in [2], see also below. Equation 1 can be written as

$$
\left[\partial_{\xi}+U, \partial_{\eta}+V\right]=0 ; \quad \partial_{\xi}=\partial / \partial \xi, \quad \partial_{\eta}=\partial / \partial \eta,
$$

which implies that this equation admits gauge transformations

i.e.

$$
\partial_{\xi}+U=g\left(\partial_{\xi}+\tilde{U}\right) g^{-1}
$$

$$
U=g \tilde{U} g^{-1}-g_{\xi} g^{-1}, \quad V=g \tilde{V} g^{-1}-g_{n} g^{-1} .
$$

With the aid of this transformation one can e.g. destroy matrices $U_{0}$ and $V_{0}$ letting $g=t^{-1}$ (see Eq. 4). After that the number of equations will be equal to the number of unknown matrices (the freedom in diagonal matrices remains). However, we will not do this transformation yet.

We do not specify here how many diagonal matrices stay free (this will become clear later). Actually, the scheme of our reasoning will be as follows: Eq. 1 will be equivalent not to one but to a set of Hamiltonian systems, each of them corresponds to a choice of undetermined diagonal matrices. Each solution of Eq. 1 is a solution of one and only one of these Hamiltonian systems. 


\section{Dressing and Undressing}

Let us fix a pole $a_{k}$. The rational function $U(z)$ can be expanded into a Taylor series in a neighborhood of this pole: $U(z)=\sum_{-\infty}^{n_{k}} U_{k, r}\left(z-a_{k}\right)^{-r-1}$; for $r \geqq 0$ the coefficients $U_{k, r}$ are the same as defined above, those for $r \leqq 0$ can be expressed as differential polynomials in elements of all the matrices $U_{i, r}, 0 \leqq r \leqq n_{i}$. The differential algebra of all such polynomials is denoted as $\mathscr{A}_{U}$ (correspondingly, $\mathscr{A}_{V}$ for the matrix $V$, and $\mathscr{A}$ for the algebra of differential polynomials in elements of both the matrices).

Proposition 2.1. There exists a gauge transformation

$$
\partial_{\xi}+U=g\left(\partial_{\xi}+A\right) g^{-1}
$$

where $g$ and $A$ are formal series in $z-a_{k}$ :

$$
g=\sum_{0}^{\infty} g_{r}\left(z-a_{k}\right)^{r}, \quad A=\sum_{-\infty}^{n_{k}} A_{r}\left(z-a_{k}\right)^{-r-1},
$$

and elements of matrices $g_{r}$ and $A_{r}$ belong to $\mathscr{A}_{U}$, such that all the matrices $A$ are diagonal.

(This time, in contrast with Eq. 6, matrices $g$ depend on z.) Equation 7 should be understood as an equality of formal series.

Proof. We perform this gauge transformation in two steps. At first, reduce the matrix $U_{k, n_{k}}$ to a diagonal form (assuming a generic case):

and let

$$
U_{k, n_{k}}=g_{0} A_{n_{k}} g_{0}^{-1}
$$

$$
\partial_{\xi}+U=g_{0}\left(\partial_{\xi}+\tilde{U}\right) g_{0}^{-1} .
$$

Now $\tilde{U}=\sum_{-\infty}^{n_{k}} \tilde{U}_{r}\left(z-a_{k}\right)^{-r-1}$, where $\tilde{U}_{n_{k}}=A_{n_{k}}$ is a diagonal matrix. Then we must solve the equation

$$
\left(\partial_{\xi}+\tilde{U}\right)=\tilde{g}\left(\partial_{\xi}+A\right) \tilde{g}^{-1},
$$

i.e. $\tilde{g}_{\xi}+\tilde{U} \tilde{g}-\tilde{g} A=0$. Substituting the series in $z-a_{k}$ one obtains a sequence of equations:

$$
\begin{array}{r}
\tilde{U}_{n_{k}} \tilde{g}_{0}-\tilde{g}_{0} A_{n_{k}}=0, \\
\tilde{U}_{n_{k}} \tilde{g}_{1}+\tilde{U}_{n_{k}-1} \tilde{g}_{0}-\tilde{g} A_{n_{k}-1}-\tilde{g}_{1} A_{n_{k}}=0, \\
\cdots \cdots \\
\tilde{g}_{0 \xi}+\tilde{U}_{n_{k}} \tilde{g}_{n_{k}+1}+\cdots+\tilde{U}_{-1} \tilde{g}_{0}-\tilde{g}_{0} A_{-1}-\cdots-\tilde{g}_{n_{k}+1} A_{n_{k}}=0 .
\end{array}
$$

Put $\tilde{g}_{0}=I$, and let the diagonals of all $\tilde{g}_{k}, k>0$ be zero. Taking the diagonal part of the second equation one can find $A_{n_{k}-1}$ while the off-diagonal part determines $\tilde{g}_{1}$ from an equation of the form $\left[A_{n_{k}}, \tilde{g}_{1}\right]=\cdots$. In a generic case, when all the diagonal elements of $A_{n_{k}}$ are distinct, this equation determines uniquely off diagonal 
elements of $\tilde{g}_{1}$. All the other matrices $A_{n_{k}-2}, \tilde{g}_{2}, \ldots$ can be determined in succession. Finally, we put $g=\tilde{g} g_{0}$.

Corollary. Matrices $g$ and $h=g^{-1}$ satisfy the equations

$$
\begin{aligned}
g_{\xi}+U g-g A & =0, \\
-h_{\xi}+h U-A h & =0,
\end{aligned}
$$

which hold as equalities for the formal series in $z-a_{k}$. (The second equation can be easily derived from the first.)

Proposition 2.2. Letting

$$
\partial_{\eta}+V=g\left(\partial_{\eta}+B\right) g^{-1}
$$

with the same $g$ as in Proposition 2.1 we obtain

$$
B=\sum_{-\infty}^{-1} B_{r}\left(z-a_{k}\right)^{-r-1}, \quad \text { if } \quad b_{k} \neq a_{k}, \quad \text { and } B=\sum_{-\infty}^{m_{k}} B_{r}\left(z-a_{k}\right)^{-r-1} \text { if } a_{k}=b_{k} \text {, }
$$

where all the $B_{r}$ are diagonal. Moreover,

$$
A_{\eta}-B_{\xi}=0
$$

Proof. We have

$$
A_{\eta}-B_{\xi}=[A, B] .
$$

For coefficients in $\left(z-a_{k}\right)^{-r-1}$ this yields

$$
A_{r, \eta}-B_{r, \xi}=\sum_{s+t=r-1}\left[A_{s}, B_{t}\right] \text {. }
$$

In this equality $B_{t}$ with the smallest number is $B_{r-1-n_{k}}$ in the term $\left[A_{n_{k}}, B_{r-1-n_{k}}\right]$. If it is already proved that all $B_{t}$ with $t>r-1-n_{k}$ are diagonal then the off-diagonal part of this commutator vanishes which proves that $B_{r-1-n_{k}}$ is also diagonal. Taking the diagonal part of the equation one obtains that $A_{\eta}-B_{\xi}=0$.

Corollary 1. Another pair of equations

$$
\begin{aligned}
g_{\eta}+V g-g B & =0 \\
-h_{\eta}+h V-B h & =0
\end{aligned}
$$

holds.

The operators $\partial_{\xi}+U, \partial_{\eta}+V$ are called "dressed", and $\partial_{\xi}+A, \partial_{\eta}+B$ "undressed." It is clear that we could write expansions in poles $b_{k}$ as well.

Corollary 2. Matrices $U_{k}$, see (2), can be represented as

$$
U_{k}=\left(g A_{-} g^{-1}\right)_{-},
$$

where the subscript - means the principal part in the expansion at the point $a_{k}$. (In this formula $g$ can be understood as a $n_{k}$-jet: only terms with $\left(\dot{z}-a_{k}\right)^{r}$ with $r \leqq n_{k}$ are involved.) If $b_{k} \neq a_{k}$, then $\left(A_{-}\right)_{\eta}=0$. If $b_{k}=a_{k}$, then $V_{k}=\left(g B_{-} g^{-1}\right)_{-}$and

$$
\left(A_{-}\right)_{\eta}-\left(B_{-}\right)_{\xi}=0 \text {. }
$$


Remark. The equality (11) is the only condition imposed on matrices $A_{-}$and $B_{-}$. Therefore we have $\min \left(n_{k}, m_{k}\right)+1$ diagonal matrices (in the case $a_{k}=b_{k}$ ) depending on both the variables $\xi$ and $\eta$ which can be freely chosen. This is the "diagonal" freedom we spoke of above.

\section{Resolvents}

Let

$$
R=g C g^{-1},
$$

where $C$ is a constant diagonal matrix, $g$ the same as above, $R$ is defined as a formal series in powers of $z-a_{k}$. $R$ is called a resolvent in the pole $a_{k}$.

Proposition 3.1. Resolvents satisfy two equations

$$
R_{\xi}=[R, U] \text { and } R_{\eta}=[R, V] \text {, }
$$

by virtue of Eq. 1 .

(Equation 1 is nothing else but a compatibility condition for these two equations.) Proof is evident if these equations are written in the form $\left[\partial_{\xi}+U, R\right]=0$, $\left[\partial_{\eta}+V, R\right]=0$ and Eq. 7 is taken into account.

Proposition 3.2. The following variational relations hold:

$$
\begin{aligned}
\delta \operatorname{tr} U_{z} R & =\operatorname{tr}(\delta U \cdot R)_{z}+\partial_{\xi}(), \\
\delta \operatorname{tr} V_{z} R & =\operatorname{tr}(\delta V \cdot R)_{z}+\partial_{\eta}(),
\end{aligned}
$$

where $\partial_{\xi}()$ and $\partial_{\eta}()$ are derivatives of 1-forms in variations $\delta U, \delta V$ and their derivatives.

\section{Corollary.}

$$
\delta \operatorname{tr} R\left(U_{z} d \xi+V_{z} d \eta\right)=\partial_{z} \operatorname{tr} R(\delta U \wedge d \xi+\delta V \wedge d \eta)+d(),
$$

where $d()$ is an exact differential of a 1-form in variations $\delta U, \delta V$ and their derivatives. Proof of the Proposition. Let us take the variation $\delta$ of Eq. 8:

$$
\begin{aligned}
\delta g_{\xi}+U \delta g+\delta U g-\delta g A-g \delta A & =0, \\
-\delta h_{\xi}+\delta h U+h \delta U-A \delta h-\delta A h & =0,
\end{aligned}
$$

and let us differentiate Eq. 8 with respect to $z$ :

$$
\begin{aligned}
g_{\xi, z}+U g_{z}+U_{z} g-g_{z} A+g A_{z} & =0, \\
-h_{\xi, z}+h_{z} U+h U_{z}-A h_{z}-A_{z} h & =0 .
\end{aligned}
$$

Multiplying the first of Eqs. 14 by $C h_{z}$, the second by $g_{z} C$, adding them and taking trace, then transforming them using integration by parts:

$$
\operatorname{tr}\left(\delta g_{\xi} C h_{z}-g_{z} C \delta h_{\xi}\right)=\partial_{\xi} \operatorname{tr}\left(\delta g C h_{z}-g_{z} C \delta h\right)+\operatorname{tr}\left(-\delta g C h_{z, \xi}+g_{z, \xi} C \delta h\right),
$$

and substituting $h_{z, \xi}$ and $g_{z, \xi}$ from Eq. 15 we obtain

$$
\operatorname{tr} \delta R U_{z}=\operatorname{tr} R_{z} \delta U-\operatorname{tr} \delta A \cdot C\left(h_{z} g+h g_{z}\right)+\operatorname{tr} C A_{z}(h \delta g+\delta h g)+\partial_{\xi}() .
$$


Terms with $A$ vanish since $h g=I$. Adding $\operatorname{tr} R \delta U_{z}$ to both the sides we get the required identity. The second equation can be proved in a similar way.

\section{Constants of Motion (First Integrals)}

Let

$$
F=\operatorname{tr} R\left(U_{z} d \xi+V_{z} d \eta\right)
$$

Proposition 4.1. The relation $d F=0$ holds by virtue of Eq. 1 .

Proof. Using Eq. 1 and Proposition 3.1 we have

$$
\begin{aligned}
d F & =\operatorname{tr}\left(\left(R_{\eta} U_{z}+R U_{z, \eta}\right) d \eta \wedge d \xi+\left(R_{\xi} V_{z}+R V_{z, \xi}\right) d \xi \wedge d \eta\right) \\
& =\operatorname{tr}\left(-[R, V] U_{z}-R U_{z, \eta}+[R, U] V_{z}+R V_{z, \xi}\right) d \xi \wedge d \eta \\
& =\operatorname{tr}\left(R\left[U_{z}, V\right]+R\left[U, V_{z}\right]-R[U, V]_{z}\right) d \xi \wedge d \eta=O
\end{aligned}
$$

Corollary. Expanding $F$ into a formal series:

$$
F=\sum_{0}^{\infty} F_{r}\left(z-a_{k}\right)^{r}
$$

we get $d F_{r}=0$ by virtue of Eq. 1 .

Definition. A constant of motion (first integral) of a partial differential equation $Q=0$ (the independent variables are $\xi_{1}, \xi_{2}, \ldots, \xi_{n}$ ) is a form

$$
f=\sum_{k}(-1)^{k-1} f_{k} d \xi_{1} \wedge \cdots \wedge d \widehat{\xi}_{k} \wedge \cdots \wedge d \xi_{n},
$$

(two such forms being identified if they coincide by virtue of the equation $Q=0$ or if they differ by an exact differential $d())$, such that

$$
d f=\sum_{k} \frac{\partial f_{k}}{\partial \xi_{k}} d \xi_{1} \wedge \cdots \wedge d \xi_{n}=0
$$

by virtue of the equation $Q=0$. (If one of the variables $\xi_{i}$ is a time variable, e.g. $\xi_{1}=t$, and others are spatial variables then the condition means that for any domain $V$

$$
\partial / \partial t \int \cdots \int_{V} f_{1} d \xi_{2} \cdots d \xi_{n}=-\int \cdots \int_{\partial V} \mathbf{f} d \sigma, \text { where } \mathbf{f}=\left(f_{2}, \ldots, f_{n}\right),
$$

i.e. $f_{1}$ is a conservative quantity and $\mathbf{f}$ is a flux.)

Thus, $F_{r}$ are constants of motion, and $F$ is their generator.

\section{Lagrangian of General Zakharov-Shabat Equations}

Here and below we use a field-theoretical Lagrange-Hamiltonian formalism (see [11]).

The suggested construction for a Lagrangian generalizes a formula given by Zakharov and Mikhailov [8], where a case of simple poles was considered. As we have seen in Sect. 2 the operator $\partial_{\xi}+U$ can be undressed in a neighborhood of 
any pole $a_{k}$ :

$$
\partial_{\xi}+U=g_{k}\left(\partial_{\xi}+A\right) g_{k}^{-1} .
$$

Here we will be interested only in principal parts of $U$ and $A$ at the point $a_{k}$, i.e. $U_{k}=\sum_{0}^{n_{k}} U_{k, r}\left(z-a_{k}\right)^{-r-1}$, and $A_{k}=\sum_{0}^{n_{k}} A_{k, r}\left(z-a_{k}\right)^{-r-1} ; g_{k}$ may be considered as a finite sum $g_{k}=\sum_{0}^{n_{k}} g_{k, r}\left(z-a_{k}\right)^{r}$, i.e. as a $n_{k}$-jet. In other words

$$
U_{k}=\left(g_{k} A_{k} g_{k}^{-1}\right)_{-}
$$

Similarly, at the points $b_{k}$

$$
V_{k}=\left(h_{k} B_{k} h_{k}^{-1}\right)_{-} .
$$

(Attention! The letter $h$ has here another meaning than in Sect. 2 where it was $g^{-1}$.) If $b_{k}=a_{k}$ then $h_{k}=g_{k}$ (one should take $\max \left(n_{k}, m_{k}\right)$-jet), see Proposition 2.2.

Now let $A_{k, r}$ be arbitrary functions of $\xi, B_{k, r}$ arbitrary functions of $\eta$ if $a_{k} \neq b_{k}$, and in the case when $a_{k}=b_{k}$ let $A_{k, r}$ and $B_{k, r}$ be arbitrary functions of $\xi$ and $\eta$ submitted to the condition $A_{k, r, \eta}-B_{k, r, \xi}=0, k \leqq \min \left(m_{k}, n_{k}\right)$. Let $U_{0}=t^{-1} t_{\xi}$, $V_{0}=t^{-1} t_{\eta}$ and let $g_{k}$ and $h_{k}$ be $n_{k}$ and $m_{k}$-jets at $a_{k}$ and $b_{k}$. If $a_{k}=b_{k}$ then $g_{k}=h_{k}$. These $g_{k}, h_{k}$ and $t$ will be variables with respect to which a Lagrangian is variated. Let

$$
\begin{aligned}
\mathscr{L}= & \operatorname{tr}\left\{\sum_{1}^{N_{1}} \operatorname{res}_{a_{k}} g_{k}^{-1}\left(\partial_{\eta}+V_{0}\right) g_{k} A_{k}-\sum_{1}^{N_{2}} \operatorname{res}_{b_{k}} h_{k}^{-1}\left(\partial_{\xi}+U_{0}\right) h_{k} B_{k}\right. \\
& \left.+\sum_{k=1}^{N_{1}} \sum_{l=1}^{N_{2}} \operatorname{res}_{a_{k}}\left(g_{k} A_{k} g_{k}^{-1}\right)_{-}\left(h_{l} B_{l} h_{l}^{-1}\right)_{-}\right\} d \xi \wedge d \eta .
\end{aligned}
$$

be the Lagrangian. (Recall [11] that, speaking more precisely, this is not a Lagrangian but an action; it is understood up to exact differentials $d($ ).)

\section{Remarks on the definition of the Lagrangian}

1. One can consider the operators $\partial_{\eta}$ and $\partial_{\xi}$ whether acting on $A_{k}$ and $B_{k}$ or not: additional terms cancel out according to the above remark.

2. In the double sum there are no terms with $a_{k}=b_{k}$, the residues of these terms vanish.

3. This formula seems to be asymmetric: the double sum contains residues at $a_{k}$ but not at $b_{k}$. Actually instead of res $a_{a_{k}}$ one can write - res $_{b_{k}}$ since if a meromorphic function has only two poles $a_{k}$ and $b_{k}$ and $f(z)=O\left(z^{-2}\right)$ when $z \rightarrow \infty$ then

$$
\operatorname{res}_{a_{k}} f(z)=-\operatorname{res}_{b_{k}} f(z) \text {. }
$$

We shall use this transformation many times.

Proposition 5.1. The variational relation

$$
\begin{aligned}
\delta \mathscr{L}= & \operatorname{tr}\left\{\sum_{1}^{N_{1}} \operatorname{res}_{a_{k}}\left[U_{k}, \partial_{\eta}+V\right] \delta g_{k} \cdot g_{k}^{-1}+\sum_{1}^{N_{2}} \operatorname{res}_{b_{k}}\left[\partial_{\xi}+U, V_{k}\right] \delta h_{k} \cdot h_{k}^{-1}\right. \\
& \left.+\left(\sum_{1}^{N_{1}} \operatorname{res}_{a_{k}}\left[U_{k}, \partial_{\eta}+V_{0}\right]+\sum_{1}^{N_{2}} \operatorname{res}_{b_{k}}\left[\partial_{\xi}+U_{0}, V_{k}\right]\right) t^{-1} \delta t\right\} \wedge d \xi \wedge d \eta-d \omega^{(1)}
\end{aligned}
$$


holds where

$$
\begin{aligned}
\omega^{(1)}= & \operatorname{tr}\left\{\sum_{1}^{N_{1}} \operatorname{res} a_{k} U_{k} d \xi \wedge \delta g_{k} \cdot g_{k}^{-1}+\sum_{1}^{N_{2}} \operatorname{res}_{b_{k}} V_{k} d \eta \wedge \delta h_{k} \cdot h_{k}^{-1}\right. \\
& \left.+\left(\sum_{1}^{N_{1}} \operatorname{res}_{a_{k}} U_{k} d \xi+\sum_{1}^{N_{2}} \operatorname{res}_{b_{k}} V_{k} d \eta\right) \wedge t^{-1} \delta t\right\}
\end{aligned}
$$

(Taking the variation we considered matrices $\left\{g_{k}\right\},\left\{h_{k}\right\}$, and $t$ (recall $U_{0}=t^{-1} t_{\xi}$, $V_{0}=t^{-1} t_{\eta}$ ) as independent variables.)

The proof can be performed by a simple calculation.

Corollary 1. The variational equations $\delta \mathscr{L} / \delta g_{k}=0, \delta \mathscr{L} / \delta h_{k}=0$, and $\delta \mathscr{L} / \delta t=0$ have the form: for any $k$

a) If $a_{k} \neq b_{k}$

$$
\left[U_{k}, \partial_{\eta}+V\right]_{-}=0, \quad\left[\partial_{\xi}+U, V_{k}\right]_{-}=0,
$$

where the subscripts minus denote principal parts at corresponding points $a_{k}$ and $b_{k}$. b) If $a_{k}=b_{k}\left(\right.$ then $g_{k}=h_{k}$ )

$$
\left[U_{k}, \partial_{\eta}+V\right]_{-}+\left[\partial_{\xi}+U, V_{k}\right]_{-}=0
$$

and one more equation

$$
\sum_{1}^{N_{1}} \operatorname{res}_{a_{k}}\left[U_{k}, \partial_{\eta}+V_{0}\right]+\sum_{1}^{N_{2}} \operatorname{res}_{b_{k}}\left[\partial_{\xi}+U_{0}, V_{k}\right]=0 .
$$

This system is equivalent to Eq. 1 .

Proof. First of all it is clear that Eq. 24 follows from the two preceding equations and gives nothing new. Equations 22, 23 express the equality for principal parts of the expansions of Eq. 1 in all the poles. Now the difference between the left-hand and right-hand sides of the equation is an entire function and zero in $z=\infty$, i.e. vanishes.

Remark. Nothing will change if we variate also the variables $A_{k}$ and $B_{k}$ as independent. It is easy to check that in this case two more equations, $g_{k, \eta}+V g_{k}=0$ and $-h_{k, \xi}+U h_{k}=0$ will appear if $a_{k} \neq b_{k}$, and an equation $g_{k, \eta}-g_{k, \xi}+V g_{k}+U h_{k}=0$ if $a_{k}=b_{k}$. Taking into account Eqs. 17, 18 we obtain (applying the transformation 20) $U_{k, \eta}=\left[U_{k}, V\right]$ and $-V_{k, \xi}=\left[U, V_{k}\right]$ if $a_{k} \neq b_{k}$ and the sum of these equations otherwise, i.e. we obtain the same equations as before.

Corollary 2. The symplectic form of Eq. 1 is $\omega=\delta \omega^{(1)}$, where $\dot{\omega}^{(1)}$ is given by Eq. 21, i.e.

$$
\begin{aligned}
\omega= & \operatorname{tr}\left\{\sum_{1}^{N_{1}} \operatorname{res}_{a_{k}}\left(\delta U_{k} \wedge d \xi \wedge \delta g_{k} \cdot g_{k}^{-1}-U_{k} d \xi \wedge \delta g_{k} \cdot g_{k}^{-1} \wedge \delta g_{k} \cdot g_{k}^{-1}\right)\right. \\
& +\sum_{1}^{N_{2}} \operatorname{res}_{b_{k}}\left(\delta V_{k} \wedge d \eta \wedge \delta h_{k} \cdot h_{k}^{-1}-V_{k} d \eta \wedge \delta h_{k} \cdot h_{k}^{-1} \wedge \delta h_{k} \cdot h_{k}^{-1}\right)
\end{aligned}
$$




$$
\begin{aligned}
& +\left(\sum_{1}^{N_{1}} \operatorname{res}_{a_{k}} \delta U_{k} \wedge d \xi+\sum_{1}^{N_{2}} \operatorname{res}_{b_{k}} \delta U_{k} \wedge d \eta\right) \wedge t^{-1} \delta t \\
& \left.+\left(\sum_{1}^{N_{1}} \operatorname{res}_{a_{k}} U_{k} d \xi+\sum_{1}^{N_{2}} \operatorname{res}_{b_{k}} V_{k} d \eta\right) \wedge t^{-1} \delta t \wedge t^{-1} \delta t\right\}
\end{aligned}
$$

Proof. See [11].

Proposition 5.2. The Hamiltonian of Eq. 1 is

$$
\mathscr{H}=-\operatorname{tr} \sum_{k=1}^{N_{1}} \sum_{l=1}^{N_{2}} \operatorname{res}_{a_{k}} U_{k} V_{l} d \xi \wedge d \eta
$$

Proof. Direct calculation according to the formula connecting the Lagrangian and Hamiltonian:

$$
\mathscr{H}=-\mathscr{L}+d \xi \wedge i\left(\tilde{\partial}_{\xi}\right) \omega^{(1)}+d \eta \wedge i\left(\tilde{\partial}_{\eta}\right) \omega^{(1)}
$$

(see [11]). Recall from [11] that we use two types of differentials: variations, $\delta f$, and $d f=\partial f / \partial \xi \cdot d \xi+\partial f / \partial \eta \cdot d \eta$. Correspondingly, there are two types of vector fields, defined by their inner products with forms. Firstly, $\partial_{\xi}$ and $\partial_{\eta}$, with $i\left(\partial_{\xi}\right) d \xi=1$, the result of substitution of this vector field into other forms being zero etc. Secondly, $\tilde{\partial}_{\xi}$ and $\tilde{\partial}_{\eta}$ with $i\left(\tilde{\partial}_{\xi}\right) \delta f=\partial f / \partial \xi$, otherwise zero.

Formula 26 is remarkably simple.

\section{Symmetries Related to Constants of Motion, Eq. 16}

Let us take a resolvent $R$ in a pole $a_{k_{0}}$. A generator of first integrals $F=$ $\operatorname{tr} R\left(U_{z} d \xi+V_{z} d \eta\right)$ was constructed whose expansion $F=\sum F_{p}\left(z-a_{k_{0}}\right)^{p}$ yields infinitely many first integrals. To every $F_{p}$ a vector field corresponds having the form

$$
\xi=\operatorname{tr}\left\{\sum_{k=1}^{N_{1}} \sum_{r=0}^{n_{k}} \sum_{(i)} \alpha_{k, r}^{(i)} \frac{\partial}{\partial g_{k, r}^{(i)}}+\sum \sum \sum \beta_{k, r}^{(i)} \frac{\partial}{\partial h_{k, r}^{(i)}}+\sum_{(i)} \gamma^{(i)} \frac{\partial}{\partial t^{(i)}}\right\} .
$$

Here we use a notation: if $a$ is a matrix then $\partial / \partial a$ is a matrix with entries: $(\partial / \partial a)_{i j}=\partial / \partial a_{j i}$. The last formula admits also a simpler representation:

$$
\xi=\operatorname{tr}\left\{\sum_{k=1}^{N_{1}} \sum_{(i)} \operatorname{res}_{a_{k}} \alpha_{k}^{(i)} \frac{\partial}{\partial g_{k}^{(i)}}+\sum_{k=1}^{N_{2}} \sum_{(i)} \operatorname{res}_{b_{k}} \beta_{k}^{(i)} \frac{\partial}{\partial h_{k}^{(i)}}+\sum_{(i)} \gamma^{(i)} \frac{\partial}{\partial t^{(i)}}\right\},
$$

where $g_{k}^{(i)}=\sum_{0}^{n_{k}} g_{k, r}^{(i)}\left(z-a_{k}\right)^{r}, \partial / \partial g_{k}^{(i)}=\sum_{r=0}^{n_{k}} \partial / \partial g_{k, r}^{(i)}\left(z-a_{k}\right)^{-r-1}, \alpha_{k}^{(i)}=\sum_{0}^{n_{k}} \alpha_{k, r}^{(i)}\left(z-a_{k}\right)^{r}$ etc.

Proposition 6.1. The vector field $\xi_{p-1}$ corresponding to $F_{p-1}$ is given by Eq. 27 with

$$
\begin{aligned}
& \alpha_{k} g_{k}^{-1}=p \sum_{r=0}^{n_{k}}\left[\left(z-a_{k}\right)^{-r-1} R\right]_{p}\left(z-a_{k}\right)^{r}, \\
& \beta_{k} h_{k}^{-1}=p \sum_{r=0}^{m_{k}}\left[\left(z-b_{k}\right)^{-r-1} R\right]_{p}\left(z-b_{k}\right)^{r}, \quad r=0,
\end{aligned}
$$


where the subscript $p$ denotes the coefficient in $\left(z-a_{k_{0}}\right)^{p}$ in the expansion in powers of $\left(z-a_{k_{0}}\right)$.

Proof. The equality

$$
\delta F_{p-1}=i\left(\xi_{p-1}\right) \omega+d()
$$

should be verified (see [11]). Equations $U_{k}=\left(g_{k} A_{k} g_{k}^{-1}\right)_{-}, V_{k}=\left(h_{k} B_{k} h_{k}^{-1}\right)_{-}$, $U_{0}=t^{-1} t_{\xi}, V_{0}=t^{-1} t_{\eta}$ imply

$$
\begin{array}{ll}
\delta U_{k}=\left[\delta g_{k} \cdot g_{k}^{-1}, U_{k}\right]_{-}, & \delta U_{0}=t^{-1} \delta t_{\xi}-t^{-1} \delta t U_{0}, \\
\delta V_{k}=\left[\delta h_{k} \cdot h_{k}^{-1}, V_{k}\right]_{-}, & \delta V_{0}=t^{-1} \delta t_{\eta}-t^{-1} \delta t V_{0}
\end{array}
$$

According to Eq. 13:

$$
\begin{aligned}
\delta F= & \partial_{z} \operatorname{tr} R(\delta U \wedge d \xi+\delta V \wedge d \eta)+d() \\
= & \partial_{z} \operatorname{tr} R\left\{\sum_{1}^{N_{1}}\left[\delta g_{k} \cdot g^{-1}, U_{k}\right]_{-} \wedge d \xi+\sum_{1}^{N_{2}}\left[\delta h_{k} \cdot h_{k}^{-1}, V_{k}\right]_{-} \wedge d \eta\right. \\
& \left.+\left(t^{-1} \delta t_{\xi}-t^{-1} \delta t U_{0}\right) \wedge d \xi+\left(t^{-1} \delta t_{\eta}-t^{-1} \delta t V_{0}\right) \wedge d \eta\right\}+d() .
\end{aligned}
$$

Using integration by parts we can replace $R t^{-1} \delta t_{\xi}$ by $R t^{-1} t_{\xi} t^{-1} \delta t-R_{\xi} t^{-1} \delta t=$ $R U_{0} t^{-1} \delta t-[R, U] t^{-1} \delta t$ and perform a similar transformation for $R t^{-1} \delta t_{\eta}$ (changing the term $d())$. We get

$$
\begin{aligned}
\delta F= & \partial_{z} \operatorname{tr} R\left\{\sum_{1}^{N_{1}}\left[\delta g_{k} \cdot g_{k}^{-1}, U_{k}\right]_{-} \wedge d \xi+\sum_{1}^{N_{2}}\left[\delta h_{k} \cdot h_{k}^{-1}, V_{k}\right]_{-} \wedge d \eta\right. \\
& \left.-\left[U-U_{0}, t^{-1} \delta t\right] \wedge d \xi-\left[V-V_{0}, t^{-1} \delta t\right] \wedge d \eta\right\}+d() .
\end{aligned}
$$

The right-hand side should be understood in the following sense. All the expressions $\left[\delta g_{k} \cdot g_{k}^{-1}, U_{k}\right]_{-}$etc. are rational functions of $z$, they can be expanded into series in $\left(z-a_{k_{0}}\right) ; R$ is such a series by definition, hence the right-hand side is such a series, too. We find the coefficient in $\left(z-a_{k_{0}}\right)^{p-1}$,

$$
\delta F_{p-1}=p \cdot \operatorname{res}_{a_{k_{0}}}\left(z-a_{k_{0}}\right)^{-p-1} \operatorname{tr} R\{\cdots\}+d(),
$$

where in the braces there is the same expression as in the preceding formula. This must coincide with $(i(\xi) \omega)_{p-1}$ where the vector field is given by Eqs. 27 and 28 , and the symplectic form by formula 25 . We find

$$
i(\xi) \delta U_{k}=i(\xi)\left[\delta g_{k} \cdot g_{k}^{-1}, U_{k}\right]_{-}=\left[\alpha_{k} g_{k}^{-1}, U_{k}\right]_{-}, \quad i(\xi) \delta V_{k}=\left[\beta_{k} h_{k}^{-1}, V_{k}\right]_{-},
$$

and taking into account that $\gamma=0$ we obtain

$$
\begin{aligned}
i(\xi) \omega= & \operatorname{tr}\left\{\sum_{1}^{N_{1}} \operatorname{res}_{a_{k}}\left(\left[\delta g_{k} \cdot g_{k}^{-1}, U_{k}\right]+\left[t^{-1} \delta t, U_{k}\right]\right) \alpha_{k} g_{k}^{-1} \wedge \delta \xi\right. \\
& \left.+\sum_{1}^{N_{2}} \operatorname{res}_{b_{k}}\left(\left[\delta h_{k} \cdot h_{k}^{-1}, V_{k}\right]+\left[t^{-1} \delta t, V_{k}\right]\right) \beta_{k} h_{k}^{-1} \wedge d \eta\right\} .
\end{aligned}
$$


Expressions 28 should be substituted into this formula, and after that one has to prove that the result coincides with Eq. 32. To this end both equations, Eq. 33 and Eq. 32, will be transformed since Eq. 33 involves residues at all the points $a_{k}, b_{k}$, and Eq. 32 at one point $a_{k_{0}}$ and it is difficult to compare them. We transform them using Eq. 20. Let us take the term with $d \xi$ of the right-hand side of Eq. 32:

$$
p \text {.tr res } a_{a_{0}}\left(z-a_{k_{0}}\right)^{-p-1} R\left[\delta g_{k} \cdot g_{k}^{-1}+t^{-1} \delta t, U_{k}\right]_{-} \wedge d \xi \text {. }
$$

Let $k \neq k_{0}$. Then in this formula $R$ can be replaced by the segment of the infinite series: $R(p)=\sum_{0}^{p} R_{r}\left(z-a_{k_{0}}\right)^{r}$. We consider this polynomial on the whole complex plane $z$. Then the above term can be transformed to

$$
-p \cdot \operatorname{tr} \operatorname{res}_{a_{k}}\left(z-a_{k_{0}}\right)^{-p-1} R(p)\left[\delta g_{k} \cdot g_{k}^{-1}+t^{-1} \delta t, U_{k}\right]_{-} \wedge d \xi .
$$

The term with $d \eta$ transforms similarly.

If $k=k_{0}$ then the corresponding term is

$$
p \cdot \operatorname{tr} \operatorname{res}_{a_{k_{0}}}\left(z-a_{k_{0}}\right)^{-p-1} R\left(p+n_{k}+1\right)\left[\delta g_{k_{0}} \cdot g_{k_{0}}^{-1}+t^{-1} \delta t, U_{k_{0}}\right]_{-} \wedge d \xi .
$$

If, in addition to this, $b_{k_{0}}=a_{k_{0}}$ then the formula will contain one more term:

$$
p \cdot \operatorname{tr} \operatorname{res}_{a_{k_{0}}}\left(z-a_{k_{0}}\right)^{-p-1} R\left(p+n_{k}+1\right)\left[\delta g_{k_{0}} \cdot g_{k_{0}}^{-1}+t^{-1} \delta t, V_{k_{0}}\right]_{-} \wedge d \eta \text {. }
$$

Now let us transform Eq. 33 taking into account Eq. 28. If $k \neq k_{0}$ then

$$
\begin{aligned}
& {\left[\left(z-a_{k}\right)^{-r-1} R\right]_{p}} \\
& \quad=\operatorname{res}_{a_{k_{0}}}\left(z-a_{k_{0}}\right)^{-p-1}\left[\left(z-a_{k}\right)^{-r-1} R\right]=\operatorname{res}_{a_{k_{0}}}\left(z-a_{k_{0}}\right)^{-p-1} R(p)\left(z-a_{k}\right)^{-r-1} \\
& \quad=-\operatorname{res}_{a_{k}}\left(z-a_{k_{0}}\right)^{-p-1} R(p)\left(z-a_{k}\right)^{-r-1}=-\left[\left(z-a_{k_{0}}\right)^{-p-1} R(p)\right]_{r},
\end{aligned}
$$

where the subscript $r$ denotes here the coefficient in the expansion in the powers of $\left(z-a_{k}\right)$ (and not of $\left.\left(z-a_{k_{0}}\right)\right)$. Then Eq. 28 becomes

$$
\alpha_{k} g_{k}^{-1}=-p\left(z-a_{k}\right)^{-p-1} R(p),
$$

where the right-hand side is understood as an expansion in the point $a_{k}$. Substituting this into Eq. 33 we obtain a term

$$
-p \cdot \operatorname{tr} \operatorname{res}_{a_{k}}\left[\delta g_{k} \cdot g_{k}^{-1}+t^{-1} \delta t, U_{k}\right]\left(z-a_{k_{0}}\right)^{-p-1} R(p) \wedge d \xi
$$

which coincides with the expression 34 . In the same way we discuss the term with $d \eta$.

If $k=k_{0}$ then Eq. 28 yields the term

$$
\alpha_{k_{0}} g_{k_{0}}^{-1}=p \sum_{r=0}^{n_{k}} R_{p+r+1}\left(z-a_{k_{0}}\right)^{r}=p\left[R\left(p+n_{k}+1\right)\left(z-a_{k_{0}}\right)^{-p-1}\right]_{+} .
$$

Substituting this into Eq. 33 we obtain exactly the term 35 . The same occurs with the term containing $d \eta$.

\section{Involutiveness of the Constants of Motion}

Proposition 7.1. The Poisson bracket of two first integrals generated by F (Eq. 16) is zero. 
Proof. For any two vector fields with $\gamma=0$ we have

$$
\begin{aligned}
\left\{F_{1}, F_{2}\right\}= & \omega\left(\xi_{1}, \xi_{2}\right) \\
= & \operatorname{tr}\left(\sum_{1}^{N_{1}} \operatorname{res}_{a_{k}}\left[\alpha_{k}^{(2)} g_{k}^{-1}, U_{k}\right] \alpha_{k}^{(1)} g_{k}^{-1} d \xi+\sum_{1}^{N_{2}} \operatorname{res}_{b_{k}}\left[\beta_{k}^{(2)} h_{k}^{-1}, V_{k}\right] \beta_{k}^{(1)} h_{k}^{-1} d \eta\right) \\
= & \operatorname{tr}\left\{\sum_{k=1}^{N_{1}} \sum_{r_{1}, r_{2}=0}^{n_{k}}\left[\left(\alpha_{k}^{(2)} g_{k}^{-1}\right)_{r_{1}} U_{k, r_{1}+r_{2}}\right]\left(\alpha_{k}^{(1)} g_{k}^{-1}\right)_{r_{2}} d \xi\right. \\
& \left.+\sum_{1}^{N_{2}} \sum_{r_{1}, r_{2}=0}^{m_{k}}\left[\left(\beta_{k}^{(2)} h_{k}^{-1}\right)_{r_{1}}, V_{k, r_{1}+r_{2}}\right]\left(\beta_{k}^{(1)} h_{k}^{-1}\right)_{r^{2}} d \eta\right\} .
\end{aligned}
$$

Let vector field $\xi_{1}$ correspond to a resolvent $R^{(1)}$ in the point $a_{k_{1}}$ and $\xi_{2}$ to resolvent $R^{(2)}$ in $a_{k_{2}}$. (If a pole $a_{k_{2}}$ is replaced by a pole $b_{k_{2}}$ the proof will stay the same.) It is sufficient to consider the generators $R^{(1)}\left(z_{1}\right)$ and $R^{(2)}\left(z_{2}\right)$ and to prove that the expression

$$
\omega\left(\xi_{1}, \xi_{2}\right)=\operatorname{tr}\left\{\sum_{k=1}^{N_{1}} \sum_{r_{1}, r_{2}=0}^{\infty}\left[\left(z_{1}-a_{k}\right)^{-r_{1}-1} R^{(1)}, U_{k, r_{1}+r_{2}}\right]\left(z_{2}-a_{k}\right)^{-r_{2}-1} R^{(2)} d \xi+\cdots\right\}
$$

(where $R^{(1)}=R^{(1)}\left(z_{1}\right), R^{(2)}=R^{(2)}\left(z_{2}\right)$, and dots mean a similar expression with $d \eta$ ) is an exact differential. Using the formula

$$
\sum_{r_{1}+r_{2}=r}\left(z_{1}-a_{k}\right)^{-r_{1}-1}\left(z_{2}-a_{k}\right)^{-r_{2}-1}=\left(z_{2}-z_{1}\right)^{-1}\left(\left(z_{1}-a_{k}\right)^{-r-1}-\left(z_{2}-a_{k}\right)^{-r-1}\right)
$$

we obtain

$$
\begin{aligned}
& \left(z_{2}-z_{1}\right) \omega\left(\xi_{1}, \xi_{2}\right) \\
& =\operatorname{tr} \sum_{k=1}^{N_{1}} \sum_{r=0}^{\infty}\left(\left[R^{(1)}, \frac{U_{k, r}}{\left(z_{1}-a_{k}\right)^{r-1}}\right] R^{(2)}-\left[R^{(1)}, \frac{U_{k, r}}{\left(z_{2}-a_{k}\right)^{r+1}}\right] R^{(2)}\right) d \xi+\cdots \\
& =\operatorname{tr}\left(\left[R^{(1)}, U\left(z_{1}\right)\right] R^{(2)}+\left[R^{(2)}, U\left(z_{2}\right)\right] R^{(1)}\right) d \xi+\cdots \\
& =\operatorname{tr}\left(R_{\xi}^{(1)} R^{(2)}+R_{\xi}^{(2)} R^{(1)}\right) d \xi+\cdots=\operatorname{tr} \partial_{\xi}\left(R^{(1)} R^{(2)}\right) d \xi+\cdots \\
& =d() .
\end{aligned}
$$

Remarks. 1. Construction of solutions of Eq. 1 is studied in [9]. 2. The idea to apply the field-theoretical formalism to integrable systems was also used by Shadwick [10].

\section{References}

1. Zakharov, V. E., Manakov, S. V., Novikov, S. P., Pitajevski, L.P.: Theory of solitons, Nauka, 1980 (Russian)

2. Dickey, L. A.: Hamiltonian structures and Lax equations generated by matrix differential operators with polynomial dependence on a parameter. Commun. Math. Phys. 88, 27-42 (1983)

3. Dickey, L. A.: Soliton equations and Hamiltonian formalism, (to be published by World Scientific)

4. Dickey, L. A.: On Hamiltonian and Lagrangian formalism for the KP-hierarchy of integrable equations. Ann. New York. Ac. Sci. 491, 131-148 (1987)

5. Gabrielov, A. M., Gelfand, I.M., Loski, M.V.: Combinatorial calculation of characteristic classes, IPM preprint 12, 1975 (Russian) 
6. Dedecker, P., Tulczyjev, W.M.: Spectral sequences and the inverse problem of the calculus of variations. Lecture notes in Mathematics vol 836, pp. 498-503 Berlin, Heidelberg, New York: Springer 1980

7. deDonder, Th.: Théorie invariantive du calcul des variationes, 1935

8. Zakharov, V. E., Mikhailov, A. V.: Variational principle for equations integrable by the inverse problem method. Funkts. Anal. Priloz., 14, 55-56 (1980)

9. Harnad, J., Saint-Aubin, Y., Shnider, S.: The soliton correlation matrix and the reduction problem for integrable systems. Commun. Math. Phys. 93, 33-56 (1984)

10. Shadwick, W. F.: The Hamilton-Cartan formalism for higher-order conserved currents. Rep. Math. Phys. 18, 243-256 (1980)

11. Dickey, L.A.: Field theoretical (multi-time) Hamiltonian formalism and its application to integrable systems. To appear in Proc. of the Annual Seminar of the Canadian Mathematical Society on Lie Theory, Differential Equations and Representation Theory, Montreal 1989

Communicated by A. Jaffe

Received May 13, 1989; in revised form December 27, 1989 
